\title{
Implante Valvular Aórtico Percutâneo: Dois Anos de Evolução da Experiência Inicial no Sul do Brasil
}

\author{
Guilherme Luiz de Melo Bernardi', Rogério Sarmento-Leite', Paulo Roberto Lunardi Prates ${ }^{1}$, \\ Alexandre Schaan de Quadros ${ }^{1}$, Imarilde Giusti ${ }^{1}$, Tailur Grando', Paulo Afonso Salgado Filho', \\ Carlos Antonio Mascia Gottschall ${ }^{1}$
}

\section{RESUMO}

Introdução: A estenose aórtica é uma afecção prevalente e com altas taxas de morbidade e mortalidade, sendo a troca valvular cirúrgica a abordagem clássica. Indivíduos idosos e com outras comorbidades apresentam elevado risco operatório. O implante valvular aórtico percutâneo surge como alternativa à cirurgia padrão. O objetivo deste estudo é apresentar o seguimento de dois anos dos primeiros casos realizados no Sul do Brasil com essa abordagem inédita. Métodos: Série de casos com descrição da técnica e resultados imediatos e a médio prazo do implante do dispositivo CoreValve ${ }^{\mathrm{TM}}$ (Medtronic Inc., Minneapolis, Estados Unidos), que consiste de uma bioprótese de pericárdio porcino montada em stent autoexpansível de nitinol, introduzido pela via arterial. Resultados: Foram submetidos ao implante desse dispositivo quatro pacientes do sexo feminino, com idade variando entre 81 anos e 90 anos e EuroSCORE logístico de $20 \%$ a $36 \%$. Observou-se significativa queda do gradiente ventrículo esquerdo-aorta, assim como ausência de complicações cardiovasculares maiores, embora duas pacientes tenham necessitado de implante de marca-passo definitivo por distúrbio de condução atrioventricular. No seguimento de dois anos observou-se melhora da classe funcional e manutenção dos gradientes e das áreas valvares aórticas alcançadas no final do procedimento e diminuição progressiva da massa ventricular esquerda. Conclusões: Esta experiência inicial com implante valvular aórtico percutâneo tem se mostrando segura e efetiva em análise a médio prazo. Estudos com seguimento a longo prazo são necessários para definir o exato papel e precisas indicações dessa nova e promissora técnica.

DESCRITORES: Estenose da valva aórtica. Próteses valvulares cardíacas. Implante de prótese de valva cardíaca.

\section{ABSTRACT}

\section{Transcatheter Aortic Valve Implantation: Two-Year Follow-up of the Initial Southern Brazilian Experience}

Background: Aortic stenosis is a prevalent disease with high morbidity and mortality, whose classical approach is surgical valve replacement. Elderly patients and those with other comorbidities present high surgical risk. Transcatheter aortic valve implantation is an effective alternative to standard surgery. The objective of this study is to report the 2-year follow-up of the first cases performed in Southern Brazil. Methods: Series of cases describing the technique, immediate and medium term results of the CoreValve ${ }^{\mathrm{TM}}$ (Medtronic Inc., Minneapolis, USA) device implantation, a porcine pericardial bioprosthesis mounted on a self expanding nitinol stent, delivered via transarterial access. Results: Four female patients, with ages ranging from 81 to 90 years and a logistic EuroSCORE ranging from $20 \%$ to $36 \%$ were successfully submitted to the implantation of this device. A significant reduction in the left ventricle-aortic gradient, and no major cardiovascular complications were observed, although 2 patients required the implantation of a permanent pacemaker due to complete atrioventricular block. Improvement of functional class and maintenance of gradients and aortic valve areas obtained at the end of the procedure, as well as a progressive decrease of left ventricular mass were observed in the 2 year follow-up. Conclusions: This early experience with the CoreValve ${ }^{\mathrm{TM}}$ transcatheter aortic valve implantation has proved to be safe and effective in the medium term outcome. Long-term follow-up studies are required to define the exact role and adequate indications for this new and promising technology.

KEY-WORDS: Aortic valve stenosis. Heart valve prosthesis. Heart valve prosthesis implantation.

\footnotetext{
1 Instituto de Cardiologia do Rio Grande do Sul - Fundação Universitária de Cardiologia (IC-FUC) - Porto Alegre, RS, Brasil.

Correspondência: Guilherme Luiz de Melo Bernardi. Av. Princesa Isabel, 395 - Porto Alegre, RS, Brasil - CEP 90620-000

E-mail: bernardi.md@gmail.com

Recebido em: 10/1/2011 • Aceito em: 4/3/2011
} 
A estenose valvar aórtica é uma das afecções cardíacas com maior prevalência em pacientes idosos. Nas últimas décadas a expectativa de vida vem aumentando e a prevalência da estenose aórtica nos octogenários hoje é próxima a 5\%. ${ }^{1,2}$ Em estágios mais avançados, a estenose aórtica apresenta elevadas taxas de morbidade e mortalidade. A cirurgia a céu aberto, com substituição valvular por prótese biológica ou metálica, é o tratamento padrão, proporcionando melhora da qualidade e maior sobrevida. ${ }^{3}$ Apesar dos bons resultados obtidos com a troca valvular cirúrgica em pacientes octogenários, ${ }^{4,5}$ muitos apresentam outras comorbidades associadas a idade, que, somadas, podem levar à mortalidade perioperatória de até $50 \%{ }^{6,7}$ Atualmente, cerca de um terço dos pacientes com estenose aórtica grave não é encaminhado para avaliação de troca valvular cirúrgica, em virtude da percepção do médico assistente de que a cirurgia cardíaca representa risco elevado. ${ }^{8}$ Assim, técnicas menos invasivas para o tratamento desse subgrupo de alto risco são necessárias.

O implante valvular aórtico percutâneo (IVAP) é uma opção de tratamento para pacientes com risco elevado para o tratamento cirúrgico convencional. Desde 2002, a partir da experiência inicial de Cribier et al. ${ }^{9}$, mais de 20 mil IVAPs, com diferentes dispositivos, já foram realizados. ${ }^{10-12}$ Essa técnica passou por evolução muito rápida, e constitui tratamento alternativo aprovado em alguns países para terapêutica em pacientes idosos e com alto risco cirúrgico. Entre os dispositivos disponíveis atualmente a prótese CoreValve ${ }^{\mathrm{TM}}$ (Medtronic Inc., Minneapolis, Estados Unidos) de pericárdio porcino montada em stent autoexpansível de nitinol está disponível para uso clínico no Brasil. Estima-se que em 2011 mais de 13 mil dessas próteses terão sido implantadas em todo o mundo. Na presente série, são descritos os resultados de dois anos de seguimento dos quatro primeiros pacientes submetidos a implante da prótese CoreValve ${ }^{\mathrm{TM}}$ em procedimentos realizados no Sul do Brasil, cuja experiência e resultados iniciais já foram previamente publicados. ${ }^{13}$

\section{MÉTODOS}

\section{Desenho do estudo e critérios de seleção}

Série de quatro casos de IVAP em pacientes com estenose aórtica grave e alto risco cirúrgico, que completaram pelo menos dois anos de seguimento clínico após a intervenção. Os procedimentos foram realizados no período de novembro de 2008 a janeiro de 2009 no Instituto de Cardiologia do Rio Grande do Sul (Porto Alegre, RS) e representam os resultados a médio prazo da prótese CoreValve ${ }^{T M}$ nessa instituição.

Os pacientes foram selecionados com base nos seguintes critérios de indicação: presença de estenose aórtica grave (área valvar $<1 \mathrm{~cm}^{2}$ ), idade $\geq 75$ anos, e alto risco cirúrgico. Era indispensável também a pre- sença de alguns dos seguintes sintomas: dor torácica ou dispneia limitante, tonturas, pré-síncope ou síncope, ou arritmia ventricular.

Todos os casos foram avaliados por uma equipe multidisciplinar composta por um cardiologista clínico, um cardiologista intervencionista e um cirurgião cardíaco, sendo a troca valvular aórtica por cirurgia considerada de alto risco para complicações pós-operatórias.

A triagem dos pacientes envolveu a avaliação de condições anatômicas e funcionais favoráveis para o implante da prótese CoreValve ${ }^{\mathrm{TM}}$. Para tanto, foram realizados ecocardiograma transtorácico com Doppler em cores, cineangiocoronariografia e angiotomografia de múltiplos detectores, conforme o caso. Os dados clínicos e angiográficos foram incluídos em formulários de acordo com o protocolo de avaliação padrão do fabricante, e a análise dessas características determinou a inclusão dos pacientes no estudo. Os pacientes elegíveis eram orientados sobre os riscos e benefícios do procedimento e assinavam o termo de consentimento livre informado.

\section{Descrição do dispositivo e do procedimento}

Foi utilizada a terceira geração da prótese valvular aórtica CoreValve ${ }^{\mathrm{TM}}$, que consiste de uma bioprótese de três folhetos de pericárdio porcino montados e suturados em stent autoexpansível de nitinol (Figura). O menor diâmetro interno da válvula tem $22 \mathrm{~mm}$ e o maior, $24 \mathrm{~mm}$, e a estrutura do stent é cortada a laser em um tubo de nitinol com $50 \mathrm{~mm}$ de comprimento. A porção distal da prótese possui alta força radial, o que permite sua expansão e a exclusão dos folhetos valvares nativos calcificados, e evita seu encolhimento pós-implante. A porção média carrega a válvula e sua arquitetura permite que os óstios coronários se mantenham livres e acessíveis. A porção proximal é alargada, permitindo sua fixação na porção proximal e estabilidade longitudinal. A válvula antes de implantada é lavada e resfriada em solução salina gelada, passa por uma

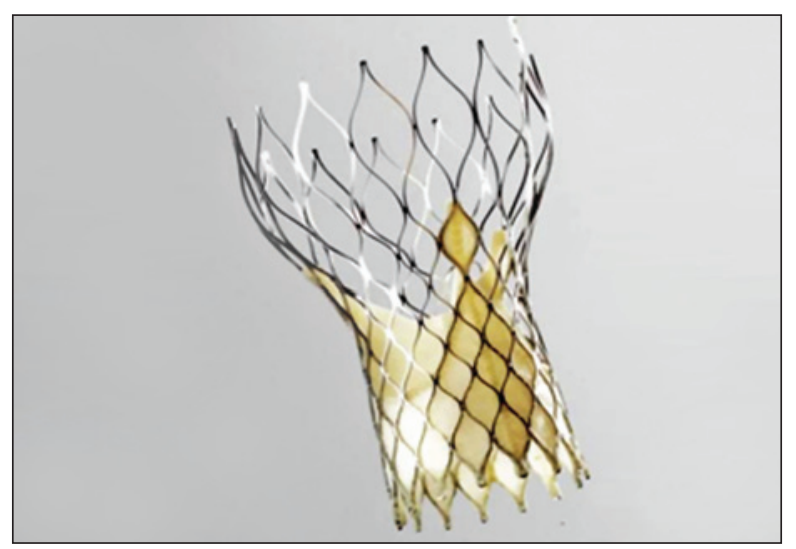

Figura - Prótese valvular aórtica CoreValve ${ }^{\mathrm{TM}}$. 
série de moldes vasculares para ter seu perfil reduzido, e é montada e fixada em um sistema de liberação de $18 \mathrm{~F}$ que entrega a prótese após valvuloplastia aórtica por balão.

Todos os pacientes receberam 200 mg de ácido acetilsalicílico (AAS) e $300 \mathrm{mg}$ de clopidogrel na noite anterior à intervenção. Os procedimentos foram realizados sob anestesia geral e em vigência de anticoagulação com heparina não-fracionada, ajustada pelo peso, para se atingir tempo de coagulação ativada de 300 segundos a 350 segundos.

Um eletrodo de marca-passo cardíaco temporário era introduzido já no início do procedimento pela veia jugular direita, para prevenir bradiarritmias e aumento da frequência cardíaca (rapid pacing) e evitar a migração do balão no momento da valvuloplastia, que precede a liberação da prótese. O acesso vascular arterial principal era obtido por dissecção da artéria femoral comum ou ilíaca ou por punção arterial, com o objetivo de se canular um vaso com pelo menos $6 \mathrm{~mm}$ de diâmetro, permitindo a introdução de uma bainha $18 \mathrm{~F}$. Outro acesso arterial no membro inferior contralateral era necessário para posicionamento de um cateter pigtail $5 \mathrm{~F}$, que serve para injeção de contraste e auxílio no momento de posicionamento e liberação da prótese.

O ventrículo esquerdo era acessado retrogradamente com um fio-guia hidrofílico de troca, introduzido através de um cateter AL1, $5 \mathrm{~F}$ ou $6 \mathrm{~F}$. Posteriormente, esse cateter era substituído por um cateter pigtail, sendo medido o gradiente transvalvar e mantendo-se o fio-guia Amplatz extrarrígido na cavidade. O balão de valvuloplastia usado, selecionado de acordo com o diâmetro do ânulo aórtico, era avançado sobre fio-guia 0.035 Amplatz do tipo extrarrígido, posicionado dentro da cavidade ventricular esquerda. Em seguida, a prótese CoreValve ${ }^{\mathrm{TM}}$, cujo tamanho era determinado previamente, era liberada.

Parâmetros clínicos e hemodinâmicos eram continuamente avaliados durante o procedimento. Aortografias contrastadas foram realizadas antes, durante e após o IVAP, para auxiliar no posicionamento e na liberação da prótese, e avaliar vazamentos ou regurgitação aórtica residual. Ecocardiografia transesofágica estava disponível e foi utilizada sempre que necessário.

Após o término dos procedimentos os pacientes eram encaminhados para uma unidade de tratamento intensivo, onde permaneciam por pelo menos 48 horas sob observação. O marca-passo temporário era mantido nesse período.

Antes da alta foi realizado ecocardiograma transtorácico e os pacientes foram orientados a manter a associação de AAS (100 mg/dia) e clopidogrel (75 mg/dia) indefinidamente.

Todos os pacientes foram reavaliados com consulta clínica, eletrocardiograma e ecocardiograma no seguimento de 1 mês, 6 meses, 1 ano e 2 anos.

\section{RESULTADOS}

Desde novembro de 2008, quando teve início esta casuística, nosso grupo já realizou 21 implantes e quatro pacientes já haviam completado pelo menos dois anos de evolução até o mês de dezembro de 2010. A Tabela 1 apresenta as características de base com dados demográficos, fatores de risco e EuroSCORE logístico dos quatro pacientes submetidos a IVAP. Todos os pacientes eram do sexo feminino, com 81 (paciente 1), 84 (paciente 2), 90 (paciente 3) e 86 (paciente 4) anos de idade e EuroSCORE logístico variando de $20 \%$ a $36 \%$. A etiologia era degenerativa em todos os casos, sendo as válvulas bastante calcificadas e com mínimo grau de regurgitação.

O acesso arterial para introdução da prótese foi realizado em duas das pacientes através de punção da artéria femoral direita e o sítio de punção foi fechado com dispositivo de sutura ProStar XL 10 (Abbott Laboratories, Abbott Park, Illinois, Estados Unidos), com acesso arterial realizado por dissecção da artéria femoral nas demais pacientes, possibilitando a introdução de bainha de $18 \mathrm{~F}$. Por questões de rotina, a primeira escolha foi a artéria femoral direita. Todos os procedimentos foram realizados com anestesia geral.

O ecocardiograma transesofágico no periprocedimento foi realizado em duas pacientes, mostrando-se útil no posicionamento adequado da prótese, na avaliação da função cardíaca durante o procedimento e na pronta avaliação do funcionamento do IVAP.

Não houve nenhum evento cardiovascular maior no período imediato pós-procedimento, e o tempo médio de permanência na unidade de tratamento intensivo foi de dois dias e de hospitalização geral, de sete dias. Em duas pacientes houve necessidade de implante de marca-passo definitivo.

\section{Resultados clínicos e laboratoriais de dois anos de seguimento}

As quatro pacientes apresentaram melhora significativa da qualidade de vida, demonstrada pela meIhora de sua classe funcional (CF), todas apresentando CF I ou II, de acordo com a classificação da New York Heart Association (NYHA). A paciente 4 vem apresentando angina estável classe II, de acordo com a classificação da Canadian Cardiovascular Society (CCS), controlada com medicação. Nenhum episódio de sincope foi relatado. A paciente 3 apresentou dois episódios de acidente vascular cerebral isquêmico (AVCi), o primeiro um mês após o IVAP e o segundo, dois anos depois, quando foi submetida a colecistectomia por videolaparoscopia em decorrência de quadro de colecistite aguda, mantendo atualmente discreta dislalia e disfagia para líquidos, mas sem perda cognitiva ou déficit funcional. As pacientes 1, 2 e 4 continuam usando AAS $100 \mathrm{mg}$ por dia, e a paciente 3 vem usando varfarina ajustada para INR 2-3 em função do quadro neurológico apresentado. Apesar da orienta- 
TABELA 1

Características de base dos pacientes submetidos ao implante valvular aórtico percutâneo

\begin{tabular}{|c|c|c|c|c|}
\hline & Paciente 1 & Paciente 2 & Paciente 3 & Paciente 4 \\
\hline Sexo & Feminino & Feminino & Feminino & Feminino \\
\hline Idade, anos & 81 & 85 & 90 & 86 \\
\hline EuroSCORE logístico, \% & 35 & 20,5 & 36 & 21,6 \\
\hline \multirow[t]{4}{*}{ Principais comorbidades } & $\begin{array}{c}\text { Hipertensão } \\
\text { arterial }\end{array}$ & $\begin{array}{l}\text { Hipertensão } \\
\text { arterial }\end{array}$ & $\begin{array}{c}\text { Cardiopatia } \\
\text { isquêmica }\end{array}$ & $\begin{array}{c}\text { Cardiopatia } \\
\text { isquêmica }\end{array}$ \\
\hline & $\begin{array}{l}\text { Cardiopatia } \\
\text { isquêmica }\end{array}$ & $\begin{array}{l}\text { Hipertensão } \\
\text { pulmonar }\end{array}$ & $\begin{array}{c}\text { Doença } \\
\text { vascular periférica }\end{array}$ & $\begin{array}{c}\text { Hipertensão } \\
\text { arterial }\end{array}$ \\
\hline & CRM prévia & Anemia & DPOC & \\
\hline & $\begin{array}{l}\text { Hipertensão } \\
\text { pulmonar }\end{array}$ & & & \\
\hline \multirow[t]{3}{*}{ Sintomas } & Angina & Angina & Angina & Angina \\
\hline & Dispneia & Dispneia & Dispneia & Dispneia \\
\hline & Tonturas & Síncope & Tonturas & Tonturas \\
\hline Classe funcional (NYHA) & IV & III & III & II \\
\hline \multirow[t]{3}{*}{ Função renal } & Normal & Normal & Normal & Normal \\
\hline & (creatinina: & (creatinina: & (creatinina: & (creatinina: \\
\hline & $0,65 \mathrm{mg} / \mathrm{dl})$ & $0,5 \mathrm{mg} / \mathrm{dl})$ & $0,9 \mathrm{mg} / \mathrm{dl})$ & $0,7 \mathrm{mg} / \mathrm{dl})$ \\
\hline Área valvular, cm² & 0,63 & 0,40 & 0,46 & 0,65 \\
\hline \multicolumn{5}{|l|}{ Gradiente VE-Ao, mmHg } \\
\hline Máximo (ecocardiograma) & 83 & 165 & 138 & 83 \\
\hline Médio (ecocardiograma) & 48 & 98 & 88 & 56 \\
\hline Pico-a-pico (hemodinâmica) & 52 & 120 & 100 & 44 \\
\hline Grau de regurgitação aórtica & Discreto & Discreto & Discreto & Discreto \\
\hline $\begin{array}{l}\text { Função ventricular e } \\
\text { fração de ejeção, \% }\end{array}$ & Normal (77) & Normal (72) & Normal (73) & Normal (73) \\
\hline
\end{tabular}

ção inicial, nenhuma paciente estava utilizando clopidogrel no segundo ano.

Nos controles eletrocardiográficos pré-implante, pósimediato e no seguimento, observou-se que uma paciente já apresentava bloqueio de ramo esquerdo (BRE) antes do IVAP (paciente 4) e as demais apresentavam somente sinais de sobrecarga ventricular esquerda. Essas três pacientes desenvolveram BRE no pós-intervenção imediato. A paciente 3 regrediu o BRE na evolução, mantendo-se em ritmo sinusal no seguimento de dois anos. As pacientes 1 e 2 desenvolveram distúrbio de condução atrioventricular avançado (bloqueio atrioventricular total) no sexto dia e no primeiro dia pós-IVAP, respectivamente. Ambas foram submetidas a implante de marca-passo definitivo. A paciente 1 apresenta, no segundo ano de seguimento, ritmo sinusal com complexo QRS estreito e marca-passo funcionando em demanda. A paciente 2 mantém ritmo de marca-passo DDD.

A avaliação ecocardiográfica no seguimento demonstrou queda sustentável do gradiente ventrículo esquerdo-aorta, manutenção da área valvar aórtica al- cançada no procedimento e diminuição progressiva da massa ventricular, demonstrados na Tabela 2. Dois anos depois do IVAP todas as pacientes apresentam funcionamento normal do dispositivo CoreValve ${ }^{T M}$ e três pacientes apresentam regurgitação aórtica mínima ao Doppler, originária de leak perivalvar.

\section{DISCUSSÃO}

Ainda não constavam na literatura nacional relatos de seguimento tardio de troca valvular aórtica pelo implante do dispositivo CoreValve ${ }^{\mathrm{TM}}$ realizados no Brasil, sendo esta experiência uma das pioneiras no meio. Nos quatro casos o implante da prótese foi realizado com resultado satisfatório, diminuindo significativamente o gradiente transvalvar aórtico, não ocorrendo eventos maiores no peroperatório e na evolução intra-hospitalar, com complicações menores tratadas e as pacientes obtendo alta em boas condições. Apesar do risco cirúrgico muito elevado, nenhuma das pacientes apresentava contraindicação formal à cirurgia cardíaca convencional. Fatores como idade acima de 80 anos, cirurgia cardíaca prévia 
TABELA 2

Seguimento ecocardiográfico antes e após implante valvular aórtico percutâneo: gradiente pressórico, área valvar aórtica e massa ventricular

\begin{tabular}{|c|c|c|c|}
\hline & Pré-implante & 1 ano & 2 anos \\
\hline \multicolumn{4}{|c|}{ Gradiente médio VE-Ao } \\
\hline Paciente 1 & $48 \mathrm{mmHg}$ & $17 \mathrm{mmHg}$ & $10 \mathrm{mmHg}$ \\
\hline Paciente 2 & $98 \mathrm{mmHg}$ & $9 \mathrm{mmHg}$ & $17 \mathrm{mmHg}$ \\
\hline Paciente 3 & $88 \mathrm{mmHg}$ & $7 \mathrm{mmHg}$ & $6 \mathrm{mmHg}$ \\
\hline Paciente 4 & $56 \mathrm{mmHg}$ & $6 \mathrm{mmHg}$ & $5 \mathrm{mmHg}$ \\
\hline \multicolumn{4}{|c|}{ Área valvar aórtica } \\
\hline Paciente 1 & $0,63 \mathrm{~cm}^{2}$ & ND & $1,4 \mathrm{~cm}^{2}$ \\
\hline Paciente 2 & $0,40 \mathrm{~cm}^{2}$ & $1,44 \mathrm{~cm}^{2}$ & $1,4 \mathrm{~cm}^{2}$ \\
\hline Paciente 3 & $0,46 \mathrm{~cm}^{2}$ & $1,54 \mathrm{~cm}^{2}$ & $1,36 \mathrm{~cm}^{2}$ \\
\hline Paciente 4 & $0,65 \mathrm{~cm}^{2}$ & $1,9 \mathrm{~cm}^{2}$ & $1,73 \mathrm{~cm}^{2}$ \\
\hline \multicolumn{4}{|c|}{ Massa ventricular } \\
\hline Paciente 1 & $124 \mathrm{~g} / \mathrm{m}^{2}$ & $107,6 \mathrm{~g} / \mathrm{m}^{2}$ & $97,2 \mathrm{~g} / \mathrm{m}^{2}$ \\
\hline Paciente 2 & $153,9 \mathrm{~g} / \mathrm{m}^{2}$ & $136,1 \mathrm{~g} / \mathrm{m}^{2}$ & $123,3 \mathrm{~g} / \mathrm{m}^{2}$ \\
\hline Paciente 3 & $179,1 \mathrm{~g} / \mathrm{m}^{2}$ & $111,3 \mathrm{~g} / \mathrm{m}^{2}$ & $81,2 \mathrm{~g} / \mathrm{m}^{2}$ \\
\hline Paciente 4 & $156 \mathrm{~g} / \mathrm{m}^{2}$ & $154 \mathrm{~g} / \mathrm{m}^{2}$ & $124,6 \mathrm{~g} / \mathrm{m}^{2}$ \\
\hline
\end{tabular}

$\mathrm{ND}=$ não disponível; $\mathrm{VE}-\mathrm{Ao}$ = ventrículo esquerdo-aorta .

e outras comorbidades associadas foram preponderantes na opção pela abordagem percutânea para o tratamento da afecção desse grupo de pacientes.

Tratamentos cirúrgicos com técnicas menos invasivas vêm sendo desenvolvidos, visando à diminuição da morbidade pós-operatória, e a opção desse novo procedimento para pacientes com alto risco cirúrgico ou para aqueles com contraindicações formais à cirurgia cardíaca vem mostrando resultados favoráveis, pelas altas taxas de sucesso e baixas taxas de complicações. ${ }^{10,11}$ Após o implante houve redução significativa do gradiente transvalvar e aumento da área valvar aórtica, que se mantiveram ao longo dos dois anos. A diminuição gradual da massa ventricular observada ao ecocardiograma reflete o remodelamento cardíaco favorável após o IVAP, dado esse que deve contribuir para melhora da qualidade de vida e aumento da sobrevida nesse grupo de pacientes. Todas as pacientes relataram ótima disposição física e melhora significativa da qualidade de vida nos últimos dois anos.

Pela sua característica de liberação, fixação e expansão, o dispositivo CoreValve ${ }^{\mathrm{TM}}$ tende a provocar certo grau de trauma ao sistema de condução elétrico do coração. A explicação teórica para tal ocorrência reside no fato de a prótese ser de nitinol e continuar seu processo de expansão no ânulo e no arco aórtico, que, associada a alterações degenerativas preexistentes no sistema de condução em pacientes dessa faixa etária, leva a bloqueios atrioventriculares e intraventricluares. Faz-se necessária a busca de preditores para a ocorrência de distúrbios de condução futuros. Como já de- monstrado ${ }^{14,15}$, e concordante com nossos achados anteriormente descritos ${ }^{16}$, o surgimento de bloqueio de ramo esquerdo após o implante desponta como um potencial marcador de risco. Porém tem-se observado que, ao longo do acompanhamento, existe a possibilidade de reversão desses bloqueios do sistema de condução ${ }^{15}$, fato esse observado em algumas das pacientes deste estudo.

Outra questão que deve ser considerada para o IVAP, e que também permanece em aberto no atual estágio do conhecimento, é a durabilidade da prótese ao longo dos anos. Enquanto a história natural a longo prazo das próteses aórticas metálicas e biológicas implantadas pela via cirúrgica convencional é conhecida, a durabilidade dos dispositivos implantáveis pela via percutânea ainda necessita ser determinada.

\section{CONCLUSÕES}

Mesmo considerando as limitações de uma série de casos com amostra pequena e ausência de grupo controle, depreende-se que a experiência inicial do Sul do Brasil com o IVAP mostrou-se segura e efetiva em análise a médio e longo prazos, com melhora dos sintomas, redução sustentável do gradiente transvalvar aórtico e manutenção da área valvar alcançada no procedimento. Estudos adicionais com maior número de pacientes, comparativos com as abordagens terapêuticas tradicionais, e de seguimento clínico mais prolongado ainda são necessários para definir o exato papel e as precisas indicações dessa nova, minimamente invasiva e promissora técnica. 


\section{CONFLITO DE INTERESSES}

Rogério Sarmento-Leite recebe apoio educacional e participa do Bureau de Palestrantes da Medtronic Brasil. Alexandre Schaan de Quadros recebe auxílio de pesquisa, apoio educacional e participa do Bureau de Palestrantes da Medtronic Brasil. Os demais autores declaram não haver conflito de interesses relacionado a este manuscrito.

\section{REFERÊNCIAS}

1. Statistical Abstract of the United States/1991. 111 th ed. Washington, DC: Department of Commerce, US Bureau of the Census; 1991.

2. Nkomo VT, Gardin JM, Skelton TN, Gottidiener JS, Scott CG, Enriquez-Sarano M. Burden of valvular heart diseases: a population-based study. Lancet. 2006;368(9540):1005-11.

3. American College of Cardiology/American Heart Association Task Force on Practice Guidelines; Society of Cardiovascular Anesthesiologists; Society for Cardiovascular Angiography and Interventions; Society of Thoracic Surgeons, Bonow RO, Carabello $B A$, Kanu $C$, de Leon $A C$ Jr, Faxon DP, Freed MD, et al. ACC/AHA 2006 guidelines for the management of patients with valvular heart disease: a report of the American College of Cardiology/American Heart Association Task Force on Practice Guidelines (writing committee to revise the 1998 Guidelines for the Management of Patients With Valvular Heart Disease): developed in collaboration with the Society of Cardiovascular Anesthesiologists: endorsed by the Society for Cardiovascular Angiography and Interventions and the Society of Thoracic Surgeons. Circulation. 2006;114(5):e84-231.

4. Thourani VH, Myung R, Kilgo P, Thompson K, Puskas JD, Latouf $\mathrm{OM}$, et al. Long-term outcomes after isolated aortic valve replacement in octogenarians: a modern perspective. Ann Thorac Surg. 2008;86(5):1458-64.

5. Chiappini B, Camurri N, Loforte A, Di Marco L, Di Bartolomeo R, Marinelli G. Outcome after aortic valve replacement in octogenarians. Ann Thorac Surg. 2004;78(1):85-9.

6. Roques F, Nashef SA, Michel P, Gauducheau E, de Vincentiis C, Baudt E, et al. Risk factors and outcome in European cardiac surgery: analysis of the EuroSCORE multinational database of 19030 patients. Eur J Cardiothorac Surg. 1999; 15(6):816-22.
7. Kvidal P, Bergstrom R, Hörte LG, Stahle E. Observed and relative survival after aortic valve replacement. J Am Coll Cardiol. 2000;35(3):747-56.

8. lung B, Baron G, Butchart EG, Delahaye F, Gohlke-Bärwolf C, Levang OW, et al. A prospective survey of patients with valvular heart disease in Europe: the Euro Heart Survey on Valvular Heart Disease. Eur Heart J. 2003;24(13):1231-43.

9. Cribier A, Eltchaninoff $H$, Bash A, Borestein N, Tron C, Bauer $\mathrm{F}$, et al. Percutaneous transcatheter implantation of an aortic valve prosthesis for calcific aortic stenosis: first human case description. Circulation. 2002;106(24):3006-8.

10. Grube E, Laborde JC, Gerckens U, Felderhoff T, Sauren B, Buellesfeld L, et al. Percutaneous implantation of the CoreValve self-expanding valve prosthesis in high-risk patients with aortic valve disease: the Siegburg first-in-man study. Circulation. 2006;114(15):1616-24.

11. Grube E, Schuler G, Buellesfeld L, Gerckens U, Linke A, Wenaweser $\mathrm{P}$, et al. Percutaneous aortic valve replacement for severe aortic stenosis in high-risk patients using the second- and current third-generation self-expanding CoreValve prosthesis: device success and 30-day clinical outcome. J Am Coll Cardiol. 2007;50(1):69-76.

12. Del Valle-Fernández R, Ruiz CE. Transcatheter heart valves for the treatment of aortic stenosis: state-of-the-art. Minerva Cardioangiol. 2008;56(5):543-56.

13. Sarmento-Leite RS, Quadros AS, Prates PRL, Zanatta LG, Salgado Filho PA, Grando T, et al. Implante valvular aórtico percutâneo: experiência inicial do Sul do Brasil. Rev Bras Cardiol Invasiva. 2008;16(4):398-405.

14. Piazza N, Onuma Y, Jesserun E, Kint PP, Maugenest AM, Anderson $\mathrm{RH}$, et al. Early and persistent intraventricular conduction abnormalities and requirements for pacemaking after percutaneous replacement of the aortic valve. JACC Cardiovasc Interv. 2008;1(3):310-6.

15. Khawaja MZ, Rajani R, Cook A, Khavandi A, Moynagh A, Chowdhary S, et al. Permanent pacemaker insertion after CoreValve transcatheter aortic valve implantation. Incidence and contributing factors (the UK CoreValve Collaborative). Circulation. 2011;123(9):951-60.

16. Sarmento-Leite RS, Quadros AS, Prates PRL, Voltolini I, Conti E, Giusti I, et al. Marca-passo permanente após implante percutâneo valvular aórtico: a necessidade é maior que imaginávamos? Rev Bras Cardiol Invasiva. 2009;17(4): 476-83. 